\title{
2018 Mental Health and Neurology Conference
}

\author{
Bangkok, Thailand; Sep 2018; Published: 25 Sep 2018 \\ Correspondence to: Dr. Moh Hasan Machfoed, Department of Neurology, Medical Faculty of Airlangga University, Surabaya 60115, \\ Indonesia. E-mail: mh.machfoed@gmail.com
}

\section{Evaluation of hypermethylation and expression pattern of glutamate and dopamine receptors genes in patients with schizophrenia}

\section{Dor Mohammad Kordi Tamandani}

\section{University of Sistan and Baluchestan}

\begin{abstract}
Aim: Schizophrenia (SCZ) is a type of psychotic disorders that affects 1\% population. Dopamine and glutamate are the major neurotransmitters in brain and their receptors are associated with the number of psychotic disorders such as schizophrenia. The aims of the present study were to analyze methylation and expression profile of dopamine and glutamate receptors genes in patients with SCZ.
\end{abstract}

Methods: Methylation-specific polymerase chain reaction (MS-PCR) was used to estimate promoter hypermethylation of dopamine and glutamate receptors genes on 81 isolated genomic DNA, from peripheral blood of individuals with schizophrenia and 71 healthy control subjects. In addition, real-time reverse transcription - PCR was used to estimate mRNA levels in 34 blood samples of healthy controls and cases.

Results: Methylation of GRM2 and GRM5, highly increased the risk of schizophrenia in comparison to reference unmethylated pattern $[\mathrm{OR}=2.82,(1.05-7.75), P=0.038],[\mathrm{OR}=12.09,(1.84-79.57), P=0.0001]$ respectively. Regarding the dopamine receptors genes, promoter methylation of $D R D 4$ and $D R D 5$ genes were statistically different $(P<0.05)$ in cases when compared with healthy controls in blood samples. Outcomes of expression analysis revealed statically significant difference between cases $(n=17)$ and health controls $(n=$ 17) regarding relative genes expression of GRM2, GRM5 and GRIA3, DRD2, DRD4 and DRD5 $(P<0.0001)$.

Conclusion: To the best of our knowledge, this is the first report which indicates the methylation status and expression profile of GRs and DRs genes with the risk of SCZ. These outcomes suggested more attention to the effect of epigenetic variations in development of SCZ in further investigations. 


\title{
2. Epilepsy: the bridge between psychiatry and neurology
}

\section{Moh Hasan Machfoed}

\author{
Airlangga University
}

Epilepsy is a group of neurological disorders characterized by epileptic seizures ${ }^{[1]}$. In 2000 BC, epilepsy was considered as a demonic possession. Hippocrates (460-370 BC) proposed that epilepsy was a medically treatable problem originating in the brain ${ }^{[2]}$. Epilepsy as a mental disorder was proposed by Morel (1857). He stated that epilepsy is caused by hereditary degeneration, which resulted in progressive intellectual and moral degeneration. The concept of epilepsy as a neurologic disorder was introduced by Robert Bentley Todd (1844) who developed the neuronal discharges theor ${ }^{[3]}$. Epilepsy reflects brain dysfunction, that can affect the mind and behavior. While the epileptic seizures themselves are episodic, the mental and behavioral changes continue, in many cases, interictally ${ }^{[4]}$. Many symptoms of neurologic or psychiatric illness - such as cognitive impairment, depression, anxiety, attention deficits, and migraine - occur more frequently in people with epilepsy than in the general population ${ }^{[5]}$. The discipline of neurology emerged from "nervous disorders" or neuropsychiatry in the late 19th century, when vascular theories of epilepsy predominated. By the turn of the 19th century psychiatry and neurology were diverging and epilepsy remained to some extent in both disciplines ${ }^{[6]}$. It was only in the middle of the 20th century with the development of electromagnetic theories of epilepsy that the concept of epilepsy per se as a neurological disorder was finally adopted in international classifications of disease. In 1960, WHO determined that epilepsy as a neurological (not mental) disease, and epilepsy with additional mental syndromes, was the province of psychiatry ${ }^{[6]}$. At the beginning of the 21st century and the centenary of the ILAE, psychiatry and neurology have been converging again, led in some respects by epilepsy, which has provided several useful models of mental illness and a bridge between psychiatry and neurology ${ }^{[6]}$.

\section{REFERENCES}

1. Chang BS, Lowenstein DH. Epilepsy. N Engl J Med 2003;349:1257-66.

2. Farrington B. Science and politics in the ancient world. New York: Barnes \& Noble; 1966. p. 64-6.

3. Binder DK, Rajneesh KF, Lee DJ, Reynolds EH. Robert Bentley Todd's contribution to cell theory and the neuron doctrine. J Hist Neurosci 2011;20:123-34

4. Korczyn AD, Schachter SC, Brodie MJ, Dalal SS, Engel J Jr, Guekht A, Hecimovic H, Jerbi K, Kanner AM, Johannessen Landmark C, Mares P, Marusic P, Meletti S, Mula M, Patsalos PN, Reuber M, Ryvlin P, Štillová K, Tuchman R, Rektor I. Epilepsy, cognition, and neuropsychiatry (Epilepsy, Brain, and Mind, part 2). Epilepsy Behav 2013;28:283-302.

5. Brooks-Kayal AR, Bath KG, Berg AT, Galanopoulou AS, Holmes GL, Jensen FE, Kanner AM, O'Brien TJ, Whittemore VH, Winawer MR, Patel M, Scharfman HE. Issues related to symptomatic and disease-modifying treatments affecting cognitive and neuropsychiatric comorbidities of epilepsy. Epilepsia 2013;54:44-60.

6. Reynolds EH, Trimble MR. Epilepsy, psychiatry, and neurology. Epilepsia 2009;50:50-5.

\section{Low level laser therapy: improved regeneration of injured sciatic nerve by $\mathrm{He}-\mathrm{Ne}$ laser}

Nasrin Takzaree, Alireza Takzaree, Nooreddin Daneshvar, Hamid Reza Foroutan

\section{Tehran University of Medical Sciences}

Neuronal tissue is one of the most important tissues of the body which recovers extremely sluggishly after being injured. Inability of the neural tissue to regenerate in response to trauma and the disability which 
ensues has prompted a lot of efforts in search for new means of neural rehabilitation. Low-energy He-Ne laser irradiation has been proposed as a sensible choice ${ }^{[1]}$. In the current experiment, sciatic nerves of 20 adult rats were crushed surgically. The subjects were randomly assigned into case and control groups. The cases received daily laser irradiation $(\lambda=65 \mathrm{~nm})$ for $4 \mathrm{~min}^{[2]}$. The muscular function of the rats was tested by angle board every 3 days, from the 3rd day postoperation. On the 27 th day, all the rats were sacrificed and the manipulated sciatic nerves were excised and studied histologically ${ }^{[3]}$. The results showed significant improvement of neural structure and muscular function in laser treated rats over the controls, as revealed by angle board testing and microscopic examination ${ }^{[4]}$. We conclude that low- energy He-Ne laser greatly restores crushed sciatic nerves in rats and can be considered for clinical trails.

\title{
REFERENCES
}

1. Mashhoudi Barez M, Tajziehchi M, Heidari MH, Bushehri A, Moayer F, Mansouri N, Safavi Naini N, Movafagh A. Stimulation effect of low level laser therapy on sciatic nerve regeneration in rat. J Lasers Med Sci 2017;8:S32-7.

2. Mester E, Mester AF, Mester A. The biomedical effects of laser application. Lasers Surg Med 1985;5:31-9.

3. Byrnes KR, Waynant RW, Ilev IK, Wu X, Barna L, Smith K, Heckert R, Gerst H, Anders JJ. Light promotes regeneration and functional recovery and alters the immune response after spinal cord injury. Lasers Surg Med 2005;36:171-85.

4. Bagis S, Comelekoglu U, Sahin G, Buyukakilli B, Erdogan C, Kanik A. Acute electrophysiologic effect of pulsed galliumarsenide low energy laser irradiation on configuration of compound nerve action potential and nerve excitability. Lasers Surg Med 2002;30:376-80.

\section{Building health research capacity in Africa for UHC: the profile of stroke in Jos, North-Central Nigeria}

\section{Godwin Osaigbovo, Ganiyu Amusa, Salaam AJ, Lucius Chidiebere Imoh, Atiene Sagay}

\author{
Jos University Teaching Hospital
}

Aim: To determine the risk factors associated with stroke, assess the case fatality 90 days post stroke, determine the bad prognostic factors of stroke and assess the sensitivity and specificity of clinical sub-typing of stroke using the WHO and Siriraj stroke scoring tools.

Methods: A longitudinal cohort study with a 90-day follow up for secondary outcome was carried out on Stroke patients admitted into the neurology unit of Jos University teaching Hospital over a 2-year period, September 1st 2016 to August 2018.

Results: A total of 246 stroke patients were admitted during the study period. Males were 131 (53.3\%) and females $115(46.6 \%)$ with an age range of $59.5 \pm 13.1$ for males and $56.7 \pm 14.2$ for females. Hypertension (81.7\%), obesity (80.9\%), dyslipidemia (54.5\%), alcohol consumption (24.8\%), carotid plaques (19.5\%), cardiac disease (19.1\%) and diabetes mellitus (18.5\%) were the commonest risk factors for stroke. The 90 days fatality for stroke was $22 \%$; however, $37 \%$ became disabled and unable to carry out activities of daily living without support. Significant predictors of mortality and morbidity were: coma, elevated glycated hemoglobin, cardiac disease, HIV infection and high National institute of health stroke score. WHO clinical stroke sub-typing showed a sensitivity of $54.3 \%$ and a specificity of $86.3 \%$ while Siriraj has a sensitivity of $87.9 \%$ and specificity of $84.9 \%$ for ischemic stroke, however, for hemorrhagic stroke, WHO sub-typing revealed a sensitivity of $86.3 \%$ and a specificity of $54.3 \%$ while Siriraj was found to have a sensitivity of $84.9 \%$ and specificity of $87.9 \%$, showing that Siriraj is a better tool for stroke categorization for appropriate management in areas where neuroimaging is neither readily available nor affordable. 
Conclusion: Stroke is a major cause of mortality and morbidity in North Central Nigeria. Community screening for risk factors should be pursued aggressively and identified risk factors managed promptly in order to reduce the burden of this pandemic. Siriraj stroke sub-typing can be used in resource limited setting like ours where neuroimaging facilities are either not available or too expensive.

\section{How to choose different treatment modalities? A retrospective study of seizure outcome in resective epilepsy surgery, vagal nerve stimulator and ketogenic diet in paediatric refractory epilepsy and their underlying etiologies; sharing experience in Tuen Mun Hospital /a regional referral center in HKSAR}

\section{Mario Wai Kwong Chak, Sui-To Wong, Fong Wai Man}

\section{Tuen Mun Hospital}

Aim: To retrospectively study effectiveness of seizure control by resective epilepsy surgery, vagal nerve stimulator and ketogenic diet/modified Atkin diet in paediatric refractory epilepsy in Tuen Mun Hospital, HKSAR and what are the patients' underlying etiologies.

Methods: Children and adolescences with medical refractory epilepsy underwent resective epilepsy surgery, vagal nerve stimulator and ketogenic diet/modified Atkin diet in Tuen Mun Hospital were recruited to study seizure outcome and underlying etiologies. We use Engel Classification to measure seizure outcome after epilepsy surgery: the seizure outcome of KD/MAD categorized into groups as: (1) seizure free; (2) $>75 \%$ seizure reduction; (3) 50\%-75\% seizure reduction; and (4) seizure no change. The seizure outcome after vagal nerve stimulator categorized into groups as: (1) > 50\% seizure reduction; and (2) Seizure no change.

Results: Forty-four patients underwent resective epilepsy surgeries from 2001 to July 2018. Age for surgery ranged from 0.8-19 years (mean 10.4 years). Follow-up duration ranged from 0.1-16 years (mean: 6.2 years) included 22 patients who underwent temporal lobe surgeries; 14 patients underwent extra-temporal lobe surgeries; 4 patients underwent hypothalamic hamartoma resection; 3 patients underwent disconnection or hemisphereotomy. Seizure outcome in respective epilepsy surgery: in temporal lobe surgeries $n=22$, Engel I: $82 \%$; Engel II: 4\%; Engel III: 8\%; Engel IV: 8\%. Etiologies: mesial temporal sclerosis: 41\%; focal cortical dysplasia: $36 \%$; developmental low grade tumor: $18 \%$; gliosis: $5 \%$. In extra-temporal lobe surgeries $n=15$, Engel I: 64\%; Engel III 36\%. Etiologies: focal cortical dysplasia: 53\%; cortical tuber 13\%; ulgyria: 7\%; haematoma/cavernous hamangioma: $13 \%$, porencephaly: $7 \%$; glial nodule: $7 \%$. In hypothalamic hamartoma surgery, Engel I: 50\%; Engel II: 25\%; Engel IV: 25\%. For disconnective surgery included 1 TPO disconnection \& 2 hemispherotomy, Engel I: 33\%; Engel II: 33\%; Engel IV: 33\%. Total 6 patients underwent vagal nerve stimulator implantation from 2014 to 2018 . Three patients underwent re-implantation after VNS out of battery. Age of first implantation $n=6$ ranged from 3-22 years (mean age: 16.5 years). Duration of follow up: ranged 1-5.5 years (mean 3.7 years).The underlying etiologies inlcuded post-FIRES epilepsy, bilateral mesial temporal sclerosis, lennox gastaut syndrome, 2 subcortical band heterotropia, symptomatic epilepsy with history of status epilepticus due to TBC1D24 mutation. Three patients had $>50 \%$ seizure reduction with etiologies included post-FIRES epilepsy, LGS, TBC1D24 mutation. Three patients with no change in seizure control with etiologies 2 subcortical band heterotropia, bilateral MTS. Twenty-one patients put on ketogenic diet/ modified Atkin diet from 2001 to July 2018. Age to start KD/MAD ranged from 0.3 year to 11 years (mean age: 5 years). Duration of follow up ranged from 2 months to 232 months (mean 32 months). Three patients became seizure free with etiologies as congenital CMV infection, Landau Kleffner Syndrome, 
suspected focal cortical dysplasia over TPO region in dominant hemisphere. Eight patients with > 75\% seizure reduction with etiologies: Leigh's disease (complex I\&IV respiratory chain enzyme deficiency), 2 suspected neuro-metabolic disease, post-HIE epilepsy, familial HLH, SSADH deficiency, post-influenza encephalopathy epilepsy, focal epilepsy. Five patients with 50\%-75\% with etiologies: Ohtahara's syndrome, post-HSV encephalitis, extensive right side fronto-central focal cortical dysplasia, 2 symptomatic generalised epilepsy. Five patients with seizure no change with etiologies: STXBP1 mutation, LGS, symptomatic epilepsy, 2 extensive focal/ hemi-cortical dysplasia, the latter two underwent epilepsy surgery with seizure improvement.

Conclusion: In view of better seizure outcome in surgery when compared with VNS and dietary treatment, refractory epilepsy patients with underlying lesion should consider epilepsy surgery. On the contrary, those with underlying metabolic or genetic disease and those with no definite focus or diffuse lesions should be considered for ketogenic diet or vagal nerve stimulator implantation.

\section{Reminiscence therapy on enhancing cognitive and improving brain wave in mild cognitive impairment}

\section{Santi Yuliani, Anita Budijanto, Dyah Wiratmi, Farida Hermani}

Prof. Dr. Soerojo Magelang Psychiatric Hospital

Aim: The aims of this research are to measure the effectiveness of reminiscence therapy on enhancing cognitive function and changes in brain waves in vascular MCI compared with MCI.

Methods: We used quasi-experimental research with pre-test and post-test study design, and statistical analysis F-test and $\chi^{2}$-test, with $\alpha=5 \%$. Subjects were divided into two groups, 16 in the MCI group and 16 in the MCI vascular group. Pre-tests were conducted prior to MOCA-INA and subjects' brain waves were examined with qEEG and brain mapping. Reminiscence therapies were conducted for 3 months for both groups and continued with the post-test.

Results: The result showed significant progress on cognitive enhancement with $P$ value $0.0002(P$ value $<$ 0.05 ) both in the MCI group and MCI vascular group. The brain wave also showed some changes particularly in the MCI group; there were some significant changes in decreasing slow wave and increasing fast wave.

Conclusion: We can conclude that reminiscence therapy is effective on enhancing cognitive and improving brain wave for mild cognitive impairment.

\section{Serum serotonin, a good indicator of insomnia in elderly with depression}

\section{Retnaningsih}

Diponegoro University/Dr. Kariadi General Hospital

Aim: To evaluate the predictive value of serum serotonin for insomnia in elderly people. 
Methods: Subjects in this retrospective study were 40 elderly people in Pucang Gading Nursing Home, Semarang, Central Java, Indonesia. Twenty-seven elderly people were diagnosed with insomnia. Serum serotonin level were measured in the elderly with and without insomnia. Bi-and multi-variate logistic regression were used to evaluate the impact of serotonin to predict insomnia.

Results: The median age of the whole population was 70 years and $90.0 \%$ were female. The elderly with serum serotonin level below $35.6 \mathrm{ng} / \mathrm{mL}$ have $\mathrm{OR}=21.600$ risk of developing insomnia compared with those with serum serotonin level more than $35.6 \mathrm{ng} / \mathrm{mL}(P=0.000)$. In bivariate analysis, low serotonin level was significantly associated with insomnia. In multivariate analysis, serotonin was an independent prognostic factor for insomnia $(P=0.001, \mathrm{OR}=0.046,95 \% \mathrm{CI})$. The AUC for serotonin was $0.846(95 \% \mathrm{CI}=0.708 \mathrm{~s} / \mathrm{d}$ $0.958 P<0.001)$.

Conclusion: We identified serum serotonin level predictive to insomnia in the elderly.

\section{Chula Stroke self-help group: a tool for empowering stroke patient}

\section{Pakkawan Vongvasinkul, Niramon Punlainak, Naruchorn Kijpaisalratana, Nijasri Charnnarong Suwanwela}

\section{King Chulalongkorn Memorial Hospital}

Aim: After acute stroke treatment in the hospital, long term post-stroke care is essential in order to prevent recurrent stroke and enhance quality of life. Stroke self-help groups engage patients, family members, and caregivers with the health care team including stroke physicians, psychiatrists, nurses, nutritionists, and social workers to help empower stroke survivors. The aim of this study is to evaluate the satisfaction among participants in the Chula Stroke Self-Help Group.

Methods: The Chula Stroke Self-Help Group has been established since 2016. The group meeting is held monthly at the outpatient department of King Chulalongkorn Memorial Hospital. The topic for each session is carefully selected by the nursing staff such as "Stroke is treatable", "Stroke is preventable", "Life after stroke", and "Stroke Rehabilitation." Each 90-min session is led by a stroke nurse, a social worker, and an invited speaker with 10-15 participants who were stroke patients, family members, or caregivers. The session begins with a brief introduction followed by extensive discussion among the participants to share their own experiences. After the session, the participants are asked to rate their satisfaction towards the activity.

Results: Thirty sessions were held between January 2016-June 2018 with a total of 351 participants. Among these, 220 (62.7\%) participants were female. Most of the participants were stroke patients (49.8\%), followed by family members of stroke patients (44.7\%) and caregivers (5.4\%). Most of the participants had a high level of satisfaction regarding self-empowerment (96.8\%), knowledge (93.7\%), and applicability to their situation (91.6\%).

Conclusion: Stroke self-help group at King Chulalongkorn Memorial Hospital can be considered as a tool for empowering stroke patients, family members, and caregivers. Participants had high satisfaction toward the activity held by the self-help group. 


\title{
9. Role of $\mathrm{S} 100 \beta$ protein as a prognostic factor in patient with acute intracerebral hemorrhage
}

\author{
Poppy Kristina Sasmita ${ }^{1}$, Ismail Setyopranoto ${ }^{2}$, Samekto Wibowo ${ }^{2}$, Ahmad Hamim Sadewa ${ }^{3}$ \\ ${ }^{1}$ Atma Jaya Catholic University of Indonesia \\ ${ }^{2}$ Universitas Gadjah Mada - Dr. Sardjito General Hospital \\ ${ }^{3}$ Universitas Gadjah Mada
}

S100 $\beta$ protein has shown its potential as biomarker of brain injury, however its efficacy is less known in intracerebral hemorrhage (ICH) cases. Patients with $\mathrm{ICH}$, who have same hematoma volume at the same location and received similar appropriate treatments, could have different neurological deficits and different prognoses. It is believed that many damages occurring in the subacute period were due to a complex pathophysiology pathway that still needs to be proven. There were 46 intracerebral hemorrhagic patients who entered the hospital consecutively during the period of August to December. CT scans were performed and met the inclusion and exclusion criteria. Neurological deficits as ascertained with National Institute of Health Stroke Scale (NIHSS). Blood samples were taken at admission to the hospital and 7th day of onset. There were 25 men (54.3\%) with a median age of 56 (31-76) years. The most important risk factor was hypertension (78.3\%). Median serum S100B levels in the ICH patients were significantly higher than those in normal individuals [22.70 (19.06-445.99) vs. $16.3(15.58-23.79) \mathrm{pg} / \mathrm{mL}, P<0.001)]$ and serum $S 100 \beta$ protein level was significantly higher in the non-survival group than in the survival group [25.86 (19.78-445.99) vs. 21.325 (19.06-63.28) $\mathrm{pg} / \mathrm{mL}, P=0.032$ ]. Serum S100 $\beta$ protein levels correlated significantly with NIHSS $(r$ $=0.418 ; P=0.004)$. Area Under Curve (AUC) of $\mathrm{S} 100 \beta$ was $0.839 \pm 0.103$ (95\% CI, 0.638-1.000), cutoff level was $28.505 \mathrm{pg} / \mathrm{mL}$ with $80 \%$ sensitivity and $87.8 \%$ specificity $(P=0.014)$. S100 $\beta$ protein levels correlated significantly with mortality within 1 week $(P=0.032)$. High levels of $S 100 \beta$ are present in the peripheral blood of patients with ICH and seem to correlate with neurological deficit and mortality within 1 week. Serum $\mathrm{S} 100 \beta$ level may be useful as marker for evaluating the prognosis of ICH.

\section{Evaluation of language dysfunction and psychological state in stroke patients}

\author{
Famida Firdose, Parvez Khandakar Ahmed, Hariprakash Chakravarthy \\ ZH Sikder Institute of Neurosciences, ZH Sikder Women's Medical College Hospital
}

Aim: Evaluate multiple faculties of language function in about 116 patients with stroke in the past 8 months.

Methods: It's an observational study that evaluated all the stroke patients who were treated at ZH Sikder Institute of Neurosciences. Excluded the patients with other comorbidity that caused dementia and language dysfunction viz, PD, Cortico basal degeneration, Alzheimer's dementia.

Results: Most of the respondents were males (90\%) ranging from 35 to 85 years. Most respondents had changeable risk factors. Significant language disturbance was seen in $30 \%$ of the patients and over $70 \%$ of the patients had minor impairment in language function. Emotional disturbance was seen in over $90 \%$ of these patients.

Conclusion: Language dysfunction is more common in stroke than we traditionally expect. Emotional 
and psychological disturbance in association with language impairment make it challenging for clinicians to treat stroke patients. Understanding the problem can lead to early detection and multidisciplinary intervention, which might be the key to good post-stroke recovery.

\title{
11. Decrease in daily dose of mestinon, methylprednisolon and level of depression affected by self motivation therapy in myasthenia gravis patients
}

\author{
Nieke Indrawati, Retnaningsih, Trianggoro Budisulistyo
}

\section{Diponegoro University Semarang}

Aim: Myasthenia gravis is an autoimmune disease ${ }^{[1]}$. One of etiologies of myasthenic crisis is emotional and physical stress ${ }^{[2]}$. We examined depression levels using the Hamilton Depression Rating Scale ${ }^{[3]}$ and the daily dose of mestinon and methilprednisolone ${ }^{[4]}$ in myasthenia gravis patients before and after 1 month of self motivation therapy.

Methods: Myasthenia gravis patients including 7 males and 18 females at Semarang Myasthenic Society were measured the level of depression with Hamilton Depression Rating Scale, daily dose of mestinon and methylprednisolon before and after 1 month self motivation therapy through motivational posters ("Thank God, I am alive and happy. Physical and emotional stress will only cause relapse of myasthenia, avoid!") that were posted in all rooms where patients could read them every day. The relationship between Hamilton Depression Rating Scale and drug dose were evaluated by using the SPSS 19.0 software. Chi square test was conducted to evaluate the categorical data. The compatibility of variables to the normal distribution was reviewed through Shapiro Wilk test. Student's t test and Wilcoxon test were used to evaluate the parametric and non parametric data. The significance limit was considered to be $P<0.05$.

Results: Hamilton score depression scale before treatment ranged from 9 to 19 , average as mild to moderate depression $(13.24 \pm 2.17)$. After being given self motivation therapy for 1 month there was a change in the hamiltonton depression scale score range from 2 to 9 , on average relatively normal (4.84 \pm 1.82$)$. There was no myasthenic crisis that occurred during the 1-month motivation period. There is a change in the dose of mestinon $(60 \mathrm{mg}$ ) daily, before therapy 2-4 times a day while post therapy 2-3 times a day, and methylprednisolone $(4 \mathrm{mg})$ daily, methilprednisolone once a day, while on therapy not used at all. Self motivational therapy has a significant effect on the Hamilton Depression Rating Scale score, changes in mestinon and methylprednisolone daily dose with $P<0.001$. Changes in depression levels affect the daily dose of mestinon $(P=0.038)$ but does not affect methylprednisolone $(P=0.371)$.

Conclusion: Reduced stress levels through self-acceptance and gratitude for life affect the level of depression and the daily dose of mestinon in myasthenia gravis patients.

\section{REFERENCES}

1. Behan WM, Behan PO. Immune complexes in myasthenia gravis. J Neurol Neurosurg Psychiatry 1979;42:595-9.

2. Beydoun SR, Wang J, Levine RL, Farvid A. Emotional stress as a trigger of myasthenic crisis and concomitant takotsubo cardiomyopathy: a case report. J Med Case Rep 2010;4:393.

3. Hamilton M. Development of a rating scale for primary depressive illness. Br J Soc Clin Psychol 1967;6:278-96.

4. Kulaksizoglu IB. Mood and anxiety disorders in patients with myasthenia gravis: aetiology, diagnosis and treatment. CNS Drugs 2007;21:473-81. 


\section{Life quality and the self in severe dementia: an intervention}

\section{Sylva Sarafidou}

\section{Center of Psychology}

The goal of psychosocial interventions in dementia is to improve the quality of life (QOL) of the sufferer. Dementia is a multifaceted disorder that affects many aspects of life and of the self. An effective intervention could target all aspects of the self, and of the disorder. The ultimate goal is to improve the QOL of the person, through enhancing the self, and all its aspects. This is challenging in severe dementia.

An intervention took place that aimed at enhancing all domains of the self, in order to improve QOL. Participants were 3 females and 2 males. All experienced individual sessions, three times per week for two months, with activities using all arts, while focusing on existing skills. The activities had a cognitive component, an emotional (expressive) one, a behavioral, and physical engagement.

These individuals had severe dementia, and no family caregivers, making assessment challenging. In order to detect the self, the I-AM test was used (requiring the completion of ten sentences starting with "I am..."), and to detect QOL, the QUALID Scale was used- an observational measure completed by the researcher and by professional caregivers.

The assessment indicated an improvement in the sense of self, and in QOL for all participants. This result can be explained psychotherapeutically and neuroscientifically: participants were brought to the "here and now" and their existing skills (and self) were enhanced, making them more functional; thus, improving their QOL, while several brain functions were combined and engaged in each session, promoting the delaying of the disorder.

Several implications arise: the self is maintained in dementia, even in the latest stage, and could be targeted in treatment, while it appears to be linked with QOL. Finally, there is a need for an intervention in severe dementia, a need to improve the living of the sufferers, and a need to focus on the abilities that remain. 